\title{
Cryptanalysis of the Modified Version of the Hash Function Proposed at PKC'98
}

\author{
Daewan Han, Sangwoo Park, and Seongtaek Chee \\ National Security Research Institute \\ 161 Gajeong-dong, Yuseong-gu, Daejeon, 305-350, Korea \\ $\{$ dwh, psw, chee\}@etri.re.kr
}

\begin{abstract}
In the conference PKC'98, Shin et al. proposed a dedicated hash function of the MD family. In this paper, we study the security of Shin's hash function. We analyze the property of the Boolean functions, the message expansion, and the data dependent rotations of the hash function. We propose a method for finding the collisions of the modified Shin's hash function and show that we can find collisions with probability $2^{-30}$.
\end{abstract}

\section{Introduction}

Hash functions are used for many cryptographic applications, such as message authentication and digital signature. A hash function is a computationally efficient function which maps binary strings of arbitrary length to binary strings of some fixed length. Cryptographic hash functions should satisfy the following properties [3]:

- pre-image resistance: given a $y$ in the image of a hash function $h$, it is computationally infeasible to find any pre-image $x$ such that $h(x)=y$.

- 2nd pre-image resistance: given $x$ and $h(x)$, it is computationally infeasible to find a $x^{\prime} \neq x$ such that $h(x)=h\left(x^{\prime}\right)$.

- collision resistance: it is computationally infeasible to find any two distinct inputs $x, x^{\prime}$ which hash to the same output.

Since the hash function MD4 [6] was introduced by R. Rivest, many dedicated hash functions based on design principles of MD4 have been proposed. MD5 [7], HAVAL [10], RIPEMD [5], RIPEMD-160 [2], and SHA-1 [4] are the dedicated hash functions of the MD family.

In the conference PKC'98, Shin et al. proposed a dedicated hash function of the MD family [8]. We call it Shin's hash function. The compression function of Shin's hash function processes a message block of 512 bits and consists of 4 rounds. Each of the rounds consists of 24 steps. Shin's hash function employs the message expansion similar to SHA-1, and Boolean functions similar to HAVAL. Another feature of Shin's hash function is to adopt the data-dependent rotations: rotations are processed by variable amounts determined by message words. 
In this paper, we study the security of Shin's hash function. We analyze the property of the Boolean functions, the message expansion, and the data dependent rotations of Shin's hash function. We indicate that, unlike the designer's intention, some of the Boolean functions of Shin's hash function fail to satisfy the Strict Avalanche Criterion(SAC) 9. Also, we point out that there can be some weakness of the message expansion and the data dependent rotations. We consider the modified Shin's hash function which is Shin's hash function whose Boolean functions all satisfy the SAC, and propose a method for finding the collisions for the modified Shin's hash function.

\section{The Compression Function of Shin's Hash Function}

Throughout this paper, the symbol + represents a modulo $2^{32}$ addition, $X \oplus Y$, $X \wedge Y$ and $X \vee Y$ represent the bitwise exclusive OR, AND, and OR of $X$ and $Y$, respectively. The symbol $X^{\ll s}$ denotes the left cyclic shift of $X$ by $s$ bit positions to the left.

The compression function of Shin's hash function processes a 16 -word message block of 512 bits, $\left(X_{0}, X_{1}, \ldots, X_{15}\right)$, and consists of 4 rounds. The 16 -word message block is expanded to a 24 -word message block, $\left(X_{0}, X_{1}, \ldots, X_{23}\right)$. In the 24-word message block, $X_{i}(i=0,1, \ldots, 15)$ are the same as the message words of the original 16-word message blocks and the additional 8 message words, $X_{i}(i=16,17, \cdots, 23)$ are determined by the 16 -word message blocks as follows:

$$
X_{16+i}=\left(X_{0+i} \oplus X_{2+i} \oplus X_{7+i} \oplus X_{12+i}\right)^{\ll 1}, i=0,1, \cdots, 7 .
$$

With the expanded 24-word message block, the compression function transforms a 5 -word(160 bits) initial value $(A, B, C, D, E)$ into a 160-bit output value. The 5 -word initial values are the followings:

$$
\begin{gathered}
A=0 x 67452301, B=0 x e f c d a b 89, C=0 \times 98 b a d c f e, \\
D=0 \times 10325476, E=0 \times c 3 d 2 e 1 f 0 .
\end{gathered}
$$

Each round of the compression function consists of 24 steps and each step processes a different word. The orders in which the words are processed differ from round to round. The word processing orders are determined by the following:

\begin{tabular}{|c|c|c|c|}
\hline Round 1 & Round 2 & Round 3 & Round 4 \\
\hline$i d$ & $\rho$ & $\rho^{2}$ & $\rho^{3}$ \\
\hline
\end{tabular}

and the permutation $\rho$ is as follows:

\begin{tabular}{|c|c|c|c|c|c|c|c|c|c|c|c|c|}
\hline$i$ & 0 & 1 & 2 & 3 & 4 & 5 & 6 & 7 & 8 & 9 & 10 & 11 \\
\hline$\rho(i)$ & 4 & 21 & 17 & 1 & 23 & 18 & 12 & 10 & 5 & 16 & 8 & 0 \\
\hline \hline$i$ & 12 & 13 & 14 & 15 & 16 & 17 & 18 & 19 & 20 & 21 & 22 & 23 \\
\hline$\rho(i)$ & 20 & 3 & 22 & 6 & 11 & 19 & 15 & 2 & 7 & 14 & 9 & 13 \\
\hline
\end{tabular}


In addition, each round employs a different constant. The constant $K_{i}(i=$ $1,2,3,4)$ is adopted by $i$-th round.

$$
K_{1}=0 \mathrm{x} 0, K_{2}=0 \times 5 a 827999, K_{3}=0 \times 6 e d 9 e b a 1, K_{4}=0 \times 8 f 1 b b c d c .
$$

In each round, one of the following Boolean functions is employed.

$$
\begin{aligned}
& f_{0}\left(x_{1}, x_{2}, x_{3}, x_{4}, x_{5}\right)=\left(x_{1} \wedge x_{2}\right) \oplus\left(x_{3} \wedge x_{4}\right) \oplus\left(x_{2} \wedge x_{3} \wedge x_{4}\right) \oplus x_{5} \\
& f_{1}\left(x_{1}, x_{2}, x_{3}, x_{4}, x_{5}\right)=x_{2} \oplus\left(\left(x_{4} \wedge x_{5}\right) \vee\left(x_{1} \wedge x_{3}\right)\right) \\
& f_{2}\left(x_{1}, x_{2}, x_{3}, x_{4}, x_{5}\right)=x_{1} \oplus\left(x_{2} \wedge\left(x_{1} \oplus x_{4}\right)\right) \oplus\left(\left(\left(x_{1} \wedge x_{4}\right) \oplus x_{3}\right) \vee x_{5}\right)
\end{aligned}
$$

The Boolean functions perform bitwise operations on words. $f_{0}, f_{1}, f_{2}, f_{0}$ are adopted by the 1st, 2nd, 3rd, and 4th round, respectively.

Now, we describe the step function of Shin's hash function. Let $T_{i, j}(j=$ $0,1, \cdots, 4)$ be the input of the step function at step $i$. Then, the step function of Shin's hash function has a transformation of the form

$$
\begin{aligned}
& T_{i, 0}=\left(f\left(T_{i, 0}, T_{i, 1}, T_{i, 2}, T_{i, 3}, T_{i, 4}\right)+X_{i}+K\right)^{\ll s}, \quad T_{i, 1}=T_{i, 1}^{\ll 10} \\
& T_{i+1,1}=T_{i, 0}, T_{i+1,2}=T_{i, 1}, T_{i+1,3}=T_{i, 2}, T_{i+1,4}=T_{i, 3}, T_{i+1,0}=T_{i, 4} .
\end{aligned}
$$

The rotation amount $s_{i}$ at the step $i$ is determined by the following:

$$
s_{i}=X_{\text {ord }(i)} \bmod 32 \text {, }
$$

where $\operatorname{ord}(i)$ is determined by the following permutations:

\begin{tabular}{|c|c|c|c|}
\hline Round 1 & Round 2 & Round 3 & Round 4 \\
\hline$\rho^{3}$ & $\rho^{2}$ & $\rho$ & $i d$ \\
\hline
\end{tabular}

\section{Some Properties of Shin's Hash Function}

In this section, we analyze the property of the Boolean functions, the message expansion, and the data dependent rotations of Shin's hash function.

\subsection{The Property of the Boolean Functions}

The designers of Shin's hash function claimed that each of the Boolean functions of the hash function is 0-1 balanced, has a high nonlinearity, and satisfies the SAC [8]. Yet, it is easy to find out that some of the Boolean functions of Shin's hash function fail to satisfy the SAC.

We define the Boolean function $f$ as satisfying the SAC if whenever one input bit of $f$ is changed, each output bit is changed with probability $1 / 2[9]$. In case of Shin's hash function, it is easy to know that the Boolean function $f_{0}$ and $f_{1}$ do not satisfy the SAC. In case of $f_{0}$, we can know that, whenever the input bit, $x_{5}$, is changed, the output bit is changed with probability 1 . Similarly, in case 
of $f_{1}$, whenever the input bit, $x_{2}$, is changed, the output bit is changed with probability 1 .

$$
\begin{aligned}
& f_{0}\left(x_{1}, x_{2}, x_{3}, x_{4}, x_{5}\right)=\left(x_{1} \wedge x_{2}\right) \oplus\left(x_{3} \wedge x_{4}\right) \oplus\left(x_{2} \wedge x_{3} \wedge x_{4}\right) \oplus x_{5} \\
& f_{1}\left(x_{1}, x_{2}, x_{3}, x_{4}, x_{5}\right)=x_{2} \oplus\left(\left(x_{4} \wedge x_{5}\right) \vee\left(x_{1} \wedge x_{3}\right)\right) .
\end{aligned}
$$

Since the designers of Shin's hash function intended that each of the Boolean functions satisfies the SAC, it can be adequate that we consider the Shin's hash function whose Boolean functions all satisfy the SAC. We call it the modified Shin's hash function.

\subsection{The Property of the Message Expansion}

The additional 8 message words $X_{16}, X_{17}, \cdots, X_{23}$ are determined by the 16 word message block by the equation (1). The equation (1) can be restated as follows:

$$
\begin{aligned}
& X_{16}=\left(X_{0} \oplus X_{2} \oplus X_{7} \oplus X_{12}\right)^{\ll 1} \\
& X_{17}=\left(X_{1} \oplus X_{3} \oplus X_{8} \oplus X_{13}\right)^{\ll 1} \\
& X_{18}=\left(X_{2} \oplus X_{4} \oplus X_{9} \oplus X_{14}\right)^{\ll 1} \\
& X_{19}=\left(X_{3} \oplus X_{5} \oplus X_{10} \oplus X_{15}\right)^{\ll 1} \\
& X_{20}=\left(X_{4} \oplus X_{6} \oplus X_{11} \oplus X_{16}\right)^{\ll 1} \\
& X_{21}=\left(X_{5} \oplus X_{7} \oplus X_{12} \oplus X_{17}\right)^{\ll 1} \\
& X_{22}=\left(X_{6} \oplus X_{8} \oplus X_{13} \oplus X_{18}\right)^{\ll 1} \\
& X_{23}=\left(X_{7} \oplus X_{9} \oplus X_{14} \oplus X_{19}\right)^{\ll 1}
\end{aligned}
$$

For two 32-bit words $X$ and $\tilde{X}$, we will define the difference of $X$ and $\tilde{X}$ as follows:

$$
\Delta X=X-\tilde{X}\left(\bmod 2^{32}\right) .
$$

To find a collision for Shin's hash function, we should find two distinct message blocks $X$ and $\tilde{X}$ which have the same hash value. In the two distinct message blocks $X$ and $\tilde{X}$, if non-zero difference occurs between $X_{i}$ and $\tilde{X}_{i}(0 \leq i \leq 15)$, non-zero difference can occur between some of the additional 8 message words which are generated from $X_{i}$ and $\tilde{X}_{i}$. For example, if non-zero difference occurs between $X_{0}$ and $\tilde{X}_{0}$, then non-zero difference can occur between $X_{16}$ and $\tilde{X}_{16}$. Furthermore, non-zero difference between $X_{16}$ and $\tilde{X}_{16}$ can make non-zero difference between $X_{20}$ and $\tilde{X}_{20}$. Thus, the message expansion can increase the difficulty of finding collisions for the hash function.

Table 1 shows the property of the message expansion of Shin's hash function. It shows that $X_{10}$ and $X_{15}$ affect $X_{19}$ and $X_{23}$ simultaneously. It means that, although $\Delta X_{10}$ and $\Delta X_{15}$ are non-zero, we can have $\Delta X_{19}=\Delta X_{23}=0$ in the case $X_{10}=X_{15}$ and $\tilde{X}_{10}=\tilde{X}_{15}$. Similarly, although $\Delta X_{17}, \Delta X_{21}$, and $\Delta X_{22}$ are non-zero, we can have $\Delta X_{8}=\Delta X_{13}=0$ in the case $X_{17} \oplus X_{21} \oplus X_{22}=0$ and $\tilde{X}_{17} \oplus \tilde{X}_{21} \oplus \tilde{X}_{22}=0$. 
Table 1. The effect of message expansion of the SHF

\begin{tabular}{|l|l|l|l|l|l|l|l|l|l|l|l|l|l|l|l|}
\hline$X_{0}$ & $X_{1}$ & $X_{2}$ & $X_{3}$ & $X_{4}$ & $X_{5}$ & $X_{6}$ & $X_{7}$ & $X_{8}$ & $X_{9}$ & $X_{10}$ & $X_{11}$ & $X_{12}$ & $X_{13}$ & $X_{14}$ & $X_{15}$ \\
\hline$X_{16}$ & $X_{17}$ & $X_{16}$ & $X_{17}$ & $X_{18}$ & $X_{19}$ & $X_{20}$ & $X_{16}$ & $X_{17}$ & $X_{18}$ & $X_{19}$ & $X_{20}$ & $X_{16}$ & $X_{17}$ & $X_{18}$ & $X_{19}$ \\
$X_{20}$ & $X_{21}$ & $X_{18}$ & $X_{19}$ & $X_{20}$ & $X_{21}$ & $X_{22}$ & $X_{20}$ & $X_{21}$ & $X_{22}$ & $X_{23}$ & & $X_{20}$ & $X_{21}$ & $X_{22}$ & $X_{23}$ \\
& & $X_{20}$ & $X_{21}$ & $X_{22}$ & $X_{23}$ & & $X_{21}$ & $X_{22}$ & $X_{23}$ & & & $X_{21}$ & $X_{22}$ & $X_{23}$ & \\
& & $X_{22}$ & $X_{23}$ & & & & $X_{23}$ & & & & & & & & \\
\hline
\end{tabular}

Now, we know that there are instances in Shin's hash function where non-zero difference between some original message words cannot be diffused to non-zero difference between some additional message words. We can use this property of the message expansion to find the collisions for the modified Shin's hash function.

\subsection{The Property of the Data Dependent Rotations}

In Shin's hash function, the data dependent rotations are adopted by the equation

$$
s_{i}=X_{\text {ord }(i)} \bmod 32,
$$

at step $i$. We can know that in the 1st round, $\operatorname{ord}(i)$ is determined by the word processing orders of the 4 th round, and $\operatorname{ord}(i)$ of the 2 nd round is determined by the word processing orders of the 3rd round, and so on.

For example, at step $1, s_{1}$ is determined by the word $X_{13}$ (see Appendix A). So, if we have the word $X_{13}$ such that $X_{13}=0 \bmod 32$, then, $s_{1}=0$. It means that we can make the data dependent rotations ineffective by choosing the appropriate message block, i.e. if we have the message block $\left(X_{i}, i=0,1, \ldots, 15\right)$ such that $X_{i}=0 \bmod 32$, we can have all $s_{i}$ equal to 0 .

Also, if we have $X_{i}$ and $\tilde{X}_{i}$ such that $\Delta X_{i} \neq 0$ and $X_{i}=\tilde{X}_{i} \bmod 32$, then we know that the shift amounts determined by $X_{i}$ and $\tilde{X}_{i}$ are the same although $X_{i}$ and $\tilde{X}_{i}$ are different.

\section{Attack on the Modified Shin's Hash Function}

Although some of the Boolean functions of Shin's hash function do not satisfy the SAC, since the designers of Shin's hash function intended that each of the Boolean functions satisfies the SAC, it seems to be adequate that we study the security of the modified Shin's hash function. In this section, we propose a method for finding the collisions for the modified Shin's hash function.

We define some notations. $A_{i}, B_{i}, C_{i}, D_{i}, E_{i}$ represent the chaining variables after step $i$ for a message block $X=\left(X_{0}, \ldots, X_{23}\right)$, and $\tilde{A}_{i}, \tilde{B}_{i}, \tilde{C}_{i}, \tilde{D}_{i}, \tilde{E}_{i}$ represent the chaining variables after step $i$ for a message block $\tilde{X}=\left(\tilde{X}_{0}, \ldots, \tilde{X}_{23}\right)$. $s_{i}$ and $\tilde{s}_{i}$ represent the shift value used in step $i$ for $X$ and $\tilde{X}$, respectively. 


\subsection{Attack on the 6 Consecutive Steps of the Modified Shin's Hash Function}

We analyze the 6 consecutive steps of the modified Shin's hash function and show how to find the collisions for 6 consecutive steps. For convenience, we consider the 6 consecutive steps from step 1 to step 6 .

To find the collisions for 6 consecutive steps, we should find two distinct message blocks $X=\left(X_{0}, X_{1}, \ldots, X_{5}\right)$ and $\tilde{X}=\left(\tilde{X}_{0}, \tilde{X}_{1}, \ldots, \tilde{X}_{5}\right)$ which have the same chaining variables after step 6, i.e. $A_{6}=\tilde{A}_{6}, B_{6}=\tilde{B}_{6}, C_{6}=\tilde{C}_{6}$, $D_{6}=\tilde{D}_{6}$, and, $E_{6}=\tilde{E}_{6}$.

We consider two distinct message blocks $X$ and $\tilde{X}$ such that $\Delta X_{0}=1 \ll 31$ and $\Delta X_{1}=\Delta X_{2}=\Delta X_{3}=\Delta X_{4}=\Delta X_{5}=0$. Then, we can have a situation that $s_{i}=\tilde{s}_{i}(i=1,2, \ldots, 6)$. We assume that $A_{0}=\tilde{A}_{0}, B_{0}=\tilde{B}_{0}, C_{0}=\tilde{C}_{0}$, $D_{0}=\tilde{D}_{0}$, and, $E_{0}=\tilde{E}_{0}$. Note that the Boolean function $f$ satisfies the SAC.

Now, we analyze each step from step 1 to step 6 and find the probability with which $(X, \tilde{X})$ can be a collision of the 6 consecutive steps. Table 2 shows the updated chaining variables at each step of the 6 consecutive steps. The boxed variables represent the updated chaining variables at each step.

Table 2. Chaining variables updated in each step

\begin{tabular}{c|ccccc|c}
\hline Step & \multicolumn{1}{|c}{$A_{0}$} & $B_{0}$ & $C_{0}$ & $D_{0}$ & $E_{0}$ & Input message \\
\hline 1 & $A_{1}$ & $B_{1}$ & $C_{1}$ & $D_{1}$ & $E_{1}$ & $X_{0}$ \\
\hline 2 & $A_{2}$ & $B_{2}$ & $C_{2}$ & $D_{2}$ & $E_{2}$ & $X_{1}$ \\
\hline 3 & $A_{3}$ & $B_{3}$ & $C_{3}$ & $D_{3}$ & $E_{3}$ & $X_{2}$ \\
\hline 4 & $A_{4}$ & $B_{4}$ & $C_{4}$ & $D_{4}$ & $E_{4}$ & $X_{3}$ \\
\hline 5 & $A_{5}$ & $B_{5}$ & $C_{5}$ & $D_{5}$ & $E_{5}$ & $X_{4}$ \\
\hline 6 & $A_{6}$ & $B_{6}$ & $C_{6}$ & $D_{6}$ & $E_{6}$ & $X_{5}$ \\
\hline
\end{tabular}

$A_{1}$ and $\tilde{A}_{1}$ are updated at step 1 as follows:

$$
\begin{array}{ll}
A_{1}=\left(f\left(A_{0}, B_{0}, C_{0}, D_{0}, E_{0}\right)+X_{0}+K\right)^{\ll s_{1}}, & B_{1}=B_{0}^{\ll 10} \\
\tilde{A}_{1}=\left(f\left(\tilde{A}_{0}, \tilde{B}_{0}, \tilde{C}_{0}, \tilde{D}_{0}, \tilde{E}_{0}\right)+\tilde{X}_{0}+K\right)^{\ll \tilde{s}_{1}}, & \tilde{B}_{1}=\tilde{B}_{0}^{\ll 10}
\end{array}
$$

Since $\Delta X_{0}=1 \ll 31$, we can have a situation that $X_{0} \oplus \tilde{X}_{0}=1 \ll 31$ with probability 1. Also, we know that $\Delta A_{0}=\Delta B_{0}=\Delta C_{0}=\Delta D_{0}=\Delta E_{0}$ and $s_{1}=\tilde{s}_{1}$. So, we have the following:

$$
A_{1} \oplus \tilde{A}_{1}=1^{\ll s_{1}\left(\text { or } \tilde{s}_{1}\right)-1} .
$$

At step 2, $E_{2}$ and $\tilde{E}_{2}$ are updated as follows:

$$
\begin{aligned}
& E_{2}=\left(f\left(E_{1}, A_{1}, B_{1}, C_{1}, D_{1}\right)+X_{1}+K\right)^{\ll s_{2}}, \quad A_{2}=A_{1}^{\ll 10}, \\
& \tilde{E}_{2}=\left(f\left(\tilde{E}_{1}, \tilde{A}_{1}, \tilde{B}_{1}, \tilde{C}_{1}, \tilde{D}_{1}\right)+\tilde{X}_{1}+K\right)^{\ll \tilde{s}_{2}}, \\
& \tilde{A}_{2}=\tilde{A}_{1}^{\ll 10} .
\end{aligned}
$$


Since $\Delta E_{1}=\Delta B_{1}=\Delta C_{1}=\Delta D_{1}=0, \Delta X_{1}=0, s_{2}=\tilde{s}_{2}$, we can have the following equation:

$$
\Delta E_{2}=0 \Longleftrightarrow f\left(E_{1}, A_{1}, B_{1}, C_{1}, D_{1}\right)=f\left(E_{1}, \tilde{A}_{1}, B_{1}, C_{1}, D_{1}\right) .
$$

Note that $A_{1} \oplus \tilde{A}_{1}=1^{\ll s_{1}\left(\text { or } \tilde{s}_{1}\right)-1}$. Since $f$ satisfies the SAC, we can have a result such that

$$
f\left(E_{1}, A_{1}, B_{1}, C_{1}, D_{1}\right)=f\left(E_{1}, \tilde{A}_{1}, B_{1}, C_{1}, D_{1}\right)
$$

with probability $1 / 2$, i.e. $\Delta E_{2}=0$ with probability $1 / 2$.

We assume that we have $\Delta E_{2}=0$ at step 2. At the next step, $D_{3}$ and $\tilde{D}_{3}$ are updated as follows:

$$
\begin{array}{ll}
D_{3}=\left(f\left(D_{2}, E_{2}, A_{2}, B_{2}, C_{2}\right)+X_{2}+K\right)^{\ll s_{3}}, & E_{3}=E_{2}^{\ll 10}, \\
\tilde{D}_{3}=\left(f\left(\tilde{D}_{2}, \tilde{E}_{2}, \tilde{A}_{2}, \tilde{B}_{2}, \tilde{C}_{2}\right)+\tilde{X}_{2}+K\right)^{\ll s_{3}}, & \tilde{E}_{3}=\tilde{E}_{2}^{\ll 10} .
\end{array}
$$

Since $\Delta D_{2}=\Delta E_{2}=\Delta B_{2}=\Delta C_{2}, \Delta X_{2}=0$, and $s_{3}=\tilde{s}_{3}$, we can have the following equation:

$$
\Delta D_{3}=0 \Longleftrightarrow f\left(D_{2}, E_{2}, A_{2}, B_{2}, C_{2}\right)=f\left(D_{2}, E_{2}, \tilde{A}_{2}, B_{2}, C_{2}\right) .
$$

Since $f$ satisfies the SAC, we can have a result such that

$$
f\left(D_{2}, E_{2}, A_{2}, B_{2}, C_{2}\right)=f\left(D_{2}, E_{2}, \tilde{A}_{2}, B_{2}, C_{2}\right)
$$

with probability $1 / 2$, i.e. $\Delta D_{3}=0$ with probability $1 / 2$.

Similarly, we can have that $\Delta C_{4}=0$ with probability $1 / 2$ at step 4 . Also, we can have that $\Delta B_{5}=0$ with probability $1 / 2$ at step 5 , and $\Delta A_{6}=0$ with probability $1 / 2$ at step 6 .

As a result, for two distinct message blocks $X=\left(X_{0}, X_{1}, \ldots, X_{5}\right)$ and $\tilde{X}=$ $\left(\tilde{X}_{0}, \tilde{X}_{1}, \ldots, \tilde{X}_{5}\right)$ such that $\Delta X_{0}=1^{\ll 31}$ and $\Delta X_{1}=\Delta X_{2}=\Delta X_{3}=\Delta X_{4}=$ $\Delta X_{5}=0$, we can have that $\Delta A_{6}=0, \Delta B_{6}=0, \Delta C_{6}=0, \Delta D_{6}=0, \Delta E_{6}=0$ with probability $2^{-5}$. So, we can find a collision for the 6 consecutive steps of the modified Shin's hash function with about $2^{5}$ operations.

\subsection{Attack on the Full Steps of the Modified Shin's Hash Function}

Now, we propose a method for finding a collision for the full steps of the modified Shin's hash function. We consider two distinct message blocks $X=\left(X_{0}, X_{1}, \ldots\right.$ ,$\left.X_{15}\right)$ and $\tilde{X}=\left(\tilde{X}_{0}, \tilde{X}_{1}, \ldots, \tilde{X}_{15}\right)$ which satisfy the following conditions.

- Condition $1: X_{i}$ is arbitrary for $i \neq 8,9$, and 10 .

- Condition $2: X_{8}=0 x 00000016$ and $X_{9}=X_{2} \oplus X_{4} \oplus X_{14} \oplus 0 x 0000000 \mathrm{~b}$

- Condition $3: X_{10}=X_{15}$

- Condition $4: \tilde{X}_{i}=X_{i}$ for $i \neq 10,15$

- Condition $5: \tilde{X}_{10}=\tilde{X}_{15}=X_{15}+1^{\ll 31}$ 
Condition 2 implies that $X_{8}=22 \bmod 32$ and $X_{18}=22 \bmod 32$. So, the shift amounts determined by $X_{8}$ and the shift amounts determined by $X_{18}$ are equal to 22 . From Condition 3,4 , and 5 , we can have that $X_{i}=\tilde{X}_{i}(i=16,17$, $\cdots, 23)$ from the property of the message expansion. Also, since $X_{i}=\tilde{X}_{i} \bmod$ $32(0 \leq i \leq 23)$, it is easy to know that $s_{i}=\tilde{s}_{i}(1 \leq i \leq 96)$. Finally, we notice that $\Delta X_{10}=\Delta X_{15}=1^{\ll 31}$.

We denote $I_{i}$ as the section of the 6 consecutive steps from step $i$ to step $i+5$ and consider the sections $I_{11}, I_{32}, I_{43}, I_{54}, I_{69}$, and, $I_{81}$. Note that the first input message word of the sections is $X_{10}$ or $X_{15}$, and $\Delta X_{10}=\Delta X_{15}=1^{\ll 31}$ (see Appendix A).

First, we consider the section $I_{32}$. We have two distinct message blocks $\left(X_{10}\right.$, $\left.X_{5}, X_{16}, X_{8}, X_{0}, X_{20}\right)$ and $\left(\tilde{X}_{10}, \tilde{X}_{5}, \tilde{X}_{16}, \tilde{X}_{8}, \tilde{X}_{0}, \tilde{X}_{20}\right)$ such that $\Delta X_{10}=1 \ll 31$ and $\Delta X_{5}=\Delta X_{16}=\Delta X_{8}=\Delta X_{0}=\Delta X_{20}=0$. So, to the section $I_{32}$, we can apply the attack on the 6 consecutive steps of the modified Shin's hash function, i.e. if $\Delta A_{31}=\Delta B_{31}=\Delta C_{31}=\Delta D_{31}=\Delta E_{31}=0$, we can have $\Delta A_{37}=\Delta B_{37}=$ $\Delta C_{37}=\Delta D_{37}=\Delta E_{37}=0$ with probability $2^{-5}$. Similarly, the attack on the 6 consecutive steps can be applied to $I_{43}, I_{54}$, and $I_{69}$ with the same probability.

However, to the section $I_{11}$, the attack on the 6 consecutive steps cannot be directly applied because $\Delta X_{15} \neq 0$, where $X_{15}$ and $\tilde{X}_{15}$ are the input message words of the last step of $I_{11}$. However, by using the property of the data dependent rotations, this problem can be solved. Note that, at step 16, $A_{16}$ and $\tilde{A}_{16}$ are updated as follows:

$$
\begin{array}{ll}
A_{16}=\left(f_{0}\left(A_{15}, B_{15}, C_{15}, D_{15}, E_{15}\right)+X_{15}+K_{1}\right)^{\ll s_{16}}, & B_{16}=B_{15}^{\ll 10} \\
\tilde{A}_{16}=\left(f_{0}\left(\tilde{A}_{15}, \tilde{B}_{15}, \tilde{C}_{15}, \tilde{D}_{15}, \tilde{E}_{15}\right)+\tilde{X}_{15}+K_{1}\right)^{\ll s_{16}}, & \tilde{B}_{15}=\tilde{B}_{15}^{\ll 10}
\end{array}
$$

We can have that $\Delta B_{15}=\Delta C_{15}=\Delta D_{15}=\Delta E_{15}=0$ with probability $2^{-4}$. Note that at step $11, A_{11}$ and $\tilde{A}_{11}$ are updated by the message words $X_{10}$ and $\tilde{X}_{10}$ such that $\Delta X_{10}=1^{\ll 31}$, and $s_{11}$ and $\tilde{s}_{11}$ are determined by $X_{18}$ and $\tilde{X}_{18}$, respectively. Since we have $X_{18}=\tilde{X}_{18}$ and $X_{18}=22 \bmod 32$ from Condition 2 , $s_{11}$ and $\tilde{s}_{11}$ are equal to 22 . Thus, we know that $A_{11} \oplus \tilde{A}_{11}=1^{\ll 21}$. At step 12 , $A_{11}$ and $\tilde{A}_{11}$ are left-rotated by 10 bit positions, i.e. $A_{12} \oplus \tilde{A}_{12}=1^{\ll 31}$. Since, from step 13 to step $15, A_{13}=A_{14}=A_{15}$ and $\tilde{A}_{13}=\tilde{A}_{14}=\tilde{A}_{15}$, the equation $A_{15} \oplus \tilde{A}_{15}=1^{\ll 31}$ holds. Now, we know that $\Delta X_{15}=\Delta A_{15}=1^{\ll 31}$, so we can have the following equation:

$$
\begin{aligned}
& \Delta A_{16}=0 \\
\Longleftrightarrow & f_{0}\left(A_{15}, B_{15}, C_{15}, D_{15}, E_{15}\right) \oplus f_{0}\left(\tilde{A}_{15}, B_{15}, C_{15}, D_{15}, E_{15}\right)=1^{\ll 31}
\end{aligned}
$$

Since $f_{0}$ satisfies the $\mathrm{SAC}$, we can have that

$$
f_{0}\left(A_{15}, B_{15}, C_{15}, D_{15}, E_{15}\right) \oplus f_{0}\left(\tilde{A}_{15}, B_{15}, C_{15}, D_{15}, E_{15}\right)=1^{\ll 31}
$$

with probability $1 / 2$. So, $\Delta A_{16}=0$ with probability $2^{-5}$. Similarly, by using the property of the data dependent rotations, this attack can be applied to the section $I_{81}$. In this case, by the value of $X_{8}$ in Condition 2, we have that $s_{81}=\tilde{s}_{81}=22$. 
As our main result, if we have the two distinct message blocks $X$ and $\tilde{X}$ which satisfy the Condition 1,2,3,4, and 5, we can find a collision of the modified Shin's hash function with probability $\left(2^{-5}\right)^{6}=2^{-30}$.

Now, we find a collision of the modified Shin's hash function by computer simulation. We employ the Boolean functions $f_{i}(i=0,1,2,3)$ which satisfy the $\mathrm{SAC}$ as follows:

$$
\begin{aligned}
& f_{0}\left(x_{1}, x_{2}, x_{3}, x_{4}, x_{5}\right)=x_{2} \oplus\left(x_{3} \wedge\left(x_{2} \oplus x_{5}\right)\right) \oplus\left(\left(\left(x_{2} \wedge x_{5}\right) \oplus x_{4}\right) \vee x_{1}\right) \\
& f_{1}\left(x_{1}, x_{2}, x_{3}, x_{4}, x_{5}\right)=x_{3} \oplus\left(x_{4} \wedge\left(x_{3} \oplus x_{1}\right)\right) \oplus\left(\left(\left(x_{3} \wedge x_{1}\right) \oplus x_{5}\right) \vee x_{2}\right) \\
& f_{2}\left(x_{1}, x_{2}, x_{3}, x_{4}, x_{5}\right)=x_{1} \oplus\left(x_{2} \wedge\left(x_{1} \oplus x_{4}\right)\right) \oplus\left(\left(\left(x_{1} \wedge x_{4}\right) \oplus x_{3}\right) \vee x_{5}\right) \\
& f_{3}\left(x_{1}, x_{2}, x_{3}, x_{4}, x_{5}\right)=x_{4} \oplus\left(x_{5} \wedge\left(x_{4} \oplus x_{2}\right)\right) \oplus\left(\left(\left(x_{4} \wedge x_{2}\right) \oplus x_{1}\right) \vee x_{3}\right)
\end{aligned}
$$

$f_{i}(i=0,1,2,3)$ is the modified version of the Boolean function $f_{2}$ of Shin's hash function, which satisfies the SAC. Note that our attack does not use the specific properties of the Boolean functions except the SAC.

As a result of computer simulation, we give a collision for the modified Shin's

\begin{tabular}{|c|c|}
\hline$X_{0}=0 x \mathrm{xe} 64 \mathrm{ec} 066$ & $\tilde{X}_{0}=0 \times \mathrm{xe} 64 \mathrm{ec} 066$ \\
\hline$X_{1}=0 x f d 126 b 95$ & $\tilde{X}_{1}=0 x f d 126 b 95$ \\
\hline$X_{2}=0 \mathrm{x} 6 \mathrm{~d} 80 \mathrm{c} 03 \mathrm{e}$ & $\tilde{X}_{2}=0 \mathrm{x} 6 \mathrm{~d} 80 \mathrm{c} 03 \mathrm{e}$ \\
\hline$X_{3}=0 \mathrm{x} 09 \mathrm{~d} 32 \mathrm{e} 0 \mathrm{c}$ & $\tilde{X}_{3}=0 \mathrm{x} 09 \mathrm{~d} 32 \mathrm{e} 0 \mathrm{c}$ \\
\hline$X_{4}=0 \times 767 \mathrm{~d} 3 \mathrm{ff} 5$ & $\tilde{X}_{4}=0 \times 767 \mathrm{~d} 3 \mathrm{ff} 5$ \\
\hline$X_{5}=0 \mathrm{x} 2 \mathrm{bc} 1 \mathrm{~b} 633$ & $\tilde{X}_{5}=0 \mathrm{x} 2 \mathrm{bc} 1 \mathrm{~b} 633$ \\
\hline$X_{6}=0 \times 40727 \mathrm{~b} 94$ & $\tilde{X}_{6}=0 \times 40727 \mathrm{~b} 94$ \\
\hline$X_{7}=0 \mathrm{xd} 7 \mathrm{e} 17540$ & $\tilde{X}_{7}=0 \mathrm{xd} 7 \mathrm{e} 17540$ \\
\hline$X_{8}=0 \mathrm{x} 00000016$ & $\tilde{X}_{8}=0 \mathrm{x} 00000016$ \\
\hline$X_{9}=0 \times 278364 \mathrm{e} 1$ & $\tilde{X}_{9}=0 \times 278364 \mathrm{e} 1$ \\
\hline$X_{10}=0 \mathrm{xe} 7 \mathrm{e} 7 \mathrm{~d} 228$ & $\tilde{X}_{10}=0 \times 67 \mathrm{e} 7 \mathrm{~d} 228$ \\
\hline$X_{11}=0 \mathrm{x} 8014 \mathrm{bf} 7 \mathrm{~d}$ & $\tilde{X}_{11}=0 \mathrm{x} 8014 \mathrm{bf} 7 \mathrm{~d}$ \\
\hline$X_{12}=0 \mathrm{xd} 5 \mathrm{a} 3 \mathrm{~b} 0 \mathrm{de}$ & $\tilde{X}_{12}=0 \mathrm{xd} 5 \mathrm{a} 3 \mathrm{~b} 0 \mathrm{de}$ \\
\hline$X_{13}=0 \times 5 a 70 f f d 6$ & $\tilde{X}_{13}=0 \times 5 \mathrm{a} 70 \mathrm{ffd} 6$ \\
\hline$X_{14}=0 \times 3 c 7 e 9 b 21$ & $\tilde{X}_{14}=0 \times 3 \mathrm{c} 7 \mathrm{e} 9 \mathrm{~b} 21$ \\
\hline$X_{15}=0 \mathrm{xe} 7 \mathrm{e} 7 \mathrm{~d} 228$ & $\tilde{X}_{15}=0 \times 67 \mathrm{e} 7 \mathrm{~d} 228$ \\
\hline
\end{tabular}
hash function in Table 3 which has the following hash value:

0xdfe4e58f, 0x1f21fb34, 0x9956457f, 0x8726dff2, 0x0a45bef3

Table 3. A collision for the modified Shin's hash function

\section{Conclusion}

In this paper, we have studied the security of Shin's hash function proposed by Shin et al. in the conference PKC'98. We have pointed out that, unlike the 
designer's intention, some of the Boolean functions of Shin's hash function do not satisfy the SAC. Also, we have indicated that there are instances in Shin's hash function that the message expansion is not effective. We have proposed a method for finding the collisions with probability $2^{-30}$ for the modified Shin's hash function. Furthermore, we have found a collision of the modified Shin's hash function by computer simulation.

Recently, the collisions of the original Shin's hash function have been found by Chang et al.. They have extended our attack, and the complexity of the attack is $2^{37}$ hashing operations [1].

This paper has provided a good example that, although it is known that the $\mathrm{SAC}$ is one of the important properties of the cryptographic Boolean functions, it can be absolutely irrelevant for the dedicated hash functions. So, we recommend that the Boolean functions of the dedicated hash function of the MD family be carefully chosen. Also, the message expansion should be carefully designed, and we conjecture that the data dependent rotations seem to be inadequate for the dedicated hash functions.

\section{References}

1. Donghoon Chang, Jaechul Sung, Soo Hak Sung, Sangjin Lee, and Jongin Lim. FullRound Differential Attack on the Hash Function Proposed at PKC'98. Proceedings of Koreacrypt'01, pages 24-35, 2002.

2. Hans Dobbertin, Antoon Bosselaers, and Bart Preneel. RIPEMD-160: A strengthened version of RIPEMD. ftp.esat.kuleuven.ac.be/pub/COSIC/bossselae/ripemd, April 1996.

3. Alfred J. Menezes, Paul C. van Oorschot, and Scott A. Vanstone. Handbook of Applied Cryptography. CRC Press, 1996.

4. National Institute of Standards and Technology. FIPS PUB 180-1 : Secure Hash Standard, April 1995.

5. Research and Development in Advanced Communications Technologies in Europe. RIPE: Integrity primitives for secure information systems. Final Report of RACE Integrity Primitives Evaluation(R1040),RACE, 1995.

6. Ronald L. Rivest. The MD4 message digest algorithm. In Alfred J. Menezes and Scott A. Vanstone, editors, Advances in Cryptology - Crypto'90, volume 537 of Lecture Notes in Computer Science, pages 303-311. Springer-Verlag, 1991.

7. Ronald L. Rivest. The MD5 message digest algorithm. In Request for Comments(RFC) 1321, April. Internet Activities Board, Internet Privacy Task Force, 1992.

8. Sang Uk Shin, Kyung Hyune Rhee, Dae Hyun Ryu, and Sang Jin Lee. A new hash function based on MDx-family and its application to MAC. In Hideki Imai and Yuliang Zheng, editors, Public Key Cryptography - PKC'98, volume 1431 of Lecture Notes in Computer Science, pages 234-246. Springer, 1998.

9. A. F. Webster and Stafford E. Tavares. On the design of S-boxes. In Hugh C. Williams, editor, Advances in Cryptology - Crypto'85, volume 218 of Lecture Notes in Computer Science, pages 523-534. Springer-Verlag, New York, 1986.

10. Yuliang Zheng, Josef Pieprzyk, and Jennifer Seberry. HAVAL-A One-Way Hashing Algorithm with Variable Length of Output. In Jennifer Seberry and Yuliang Zheng, editors, Advances in Cryptology - Auscrypt'92, volume 718 of Lecture Notes in Computer Science, pages 83-104. Springer, 1992. 
A Message Processing Orders of Shin's Hash Function

\begin{tabular}{|c|c||c|c||c|c||c|c|}
\hline Step & Word & Step & Word & Step & Word & Step & Word \\
\hline 1 & $X_{0}$ & 25 & $X_{4}$ & 49 & $X_{23}$ & 73 & $X_{13}$ \\
2 & $X_{1}$ & 26 & $X_{21}$ & 50 & $X_{14}$ & 74 & $X_{22}$ \\
3 & $X_{2}$ & 27 & $X_{17}$ & 51 & $X_{19}$ & 75 & $X_{2}$ \\
4 & $X_{3}$ & 28 & $X_{1}$ & 52 & $X_{21}$ & 76 & $X_{14}$ \\
5 & $X_{4}$ & 29 & $X_{23}$ & 53 & $X_{13}$ & 77 & $X_{3}$ \\
6 & $X_{5}$ & 30 & $X_{18}$ & 54 & $X_{15}$ & 78 & $X_{6}$ \\
7 & $X_{6}$ & 31 & $X_{12}$ & 55 & $X_{20}$ & 79 & $X_{7}$ \\
8 & $X_{7}$ & 32 & $X_{10}$ & 56 & $X_{8}$ & 80 & $X_{5}$ \\
9 & $X_{8}$ & 33 & $X_{5}$ & 57 & $X_{18}$ & 81 & $X_{15}$ \\
10 & $X_{9}$ & 34 & $X_{16}$ & 58 & $X_{11}$ & 82 & $X_{0}$ \\
11 & $X_{10}$ & 35 & $X_{8}$ & 59 & $X_{5}$ & 83 & $X_{18}$ \\
\cline { 2 - 5 } 12 & $X_{11}$ & 36 & $X_{0}$ & 60 & $X_{4}$ & 84 & $X_{23}$ \\
13 & $X_{12}$ & 37 & $X_{20}$ & 61 & $X_{7}$ & 85 & $X_{10}$ \\
14 & $X_{13}$ & 38 & $X_{3}$ & 62 & $X_{1}$ & 86 & $X_{21}$ \\
15 & $X_{14}$ & 39 & $X_{22}$ & 63 & $X_{9}$ & 87 & $X_{16}$ \\
16 & $X_{15}$ & 40 & $X_{6}$ & 64 & $X_{12}$ & 88 & $X_{20}$ \\
17 & $X_{16}$ & 41 & $X_{11}$ & 65 & $X_{0}$ & 89 & $X_{4}$ \\
18 & $X_{17}$ & 42 & $X_{19}$ & 66 & $X_{2}$ & 90 & $X_{17}$ \\
19 & $X_{18}$ & 43 & $X_{15}$ & 67 & $X_{6}$ & 91 & $X_{12}$ \\
20 & $X_{19}$ & 44 & $X_{2}$ & 68 & $X_{17}$ & 92 & $X_{19}$ \\
21 & $X_{20}$ & 45 & $X_{7}$ & 69 & $X_{10}$ & 93 & $X_{8}$ \\
22 & $X_{21}$ & 46 & $X_{14}$ & 70 & $X_{22}$ & 94 & $X_{9}$ \\
23 & $X_{22}$ & 47 & $X_{9}$ & 71 & $X_{16}$ & 95 & $X_{14}$ \\
24 & $X_{23}$ & 48 & $X_{13}$ & 72 & $X_{3}$ & 96 & $X_{1}$ \\
\hline
\end{tabular}

